\title{
A novel wave-energy device with enhanced wave amplification and induction actuator
}

\author{
Onno Bokhove, Anna Kalogirou, David Henry, and Gareth P. Thomas
}

\begin{abstract}
A novel wave-energy device is presented. Both a preliminary proof-of-principle of a working, scaled laboratory version of the energy device is shown as well as the derivation and analysis of a comprehensive mathematical and numerical model of the new device. The wave-energy device includes a convergence in which the waves are amplified, a constrained wave buoy with a (curved) mast and direct energy conversion of the buoy motion into electrical power via an electro-magnetic generator. The device is designed for use in breakwaters and it is possible to be taken out of action during severe weather. The new design is a deconstruction of elements of existing waveenergy devices, such as the TapChan, IP wave-buoy and the Berkeley Wedge, put together in a different manner to enhance energy conversion and, hence, efficiency. The idea of wave-focusing in a contraction emerged from our work on creating and simulating rogue waves in crossing seas, including a "bore-soliton-splash". Such crossing seas have been recreated and modelled in the laboratory and in simulations by using a geometric channel convergence. The mathematical and numerical modelling is also novel. One monolithic variational principle governs the dynamics including the combined (potential-flow) hydrodynamics, the buoy motion and the power generation, to which the dissipative elements such as the electrical resistance of the circuits, coils and loads have been added $a$ posteriori. The numerical model is a direct and consistent discretisation of this comprehensive variational principle. Preliminary numerical calculations are shown for the case of linearised dynamics; optimisation of efficiency is a target of future work.
\end{abstract}

Index Terms-water-wave focussing, wave-energy buoy, electro-magnetic generator, monolithic variational principle, finite-element modelling

\section{INTRODUCTION}

$\mathrm{M}$ OTIVATED by previous research on shallowwater and shallow-granular flows through contracting channels, and by water-wave impact against sea walls, a series of $a d-h o c$ experiments have been

Manuscript received 16 March; accepted 27 March; published 12 May, 2020. This is an open access article distributed under the terms of the Creative Commons Attribution 4.0 licence (CC BY http://creativecommons.org/licenses/by/4.0/). unrestricted use (including commercial), distribution and reproduction is permitted provided that credit is given to the original author(s) of the work, including a URI or hyperlink to the work, this public license and a copyright notice. This article has been subject to single-blind peer review by a minimum of two reviewers. This is follow-up research that grew out of EPSRC grant EP/L025388/1 for O. Bokhove and A. Kalogirou.

O. Bokhove: School of Mathematics, University of Leeds, LS2 9JT, Leeds, UK; o.bokhove@leeds.ac.uk

A. Kalogirou: School of Mathematical Sciences, University of Nottingham, NG7 2RD, Nottingham, UK; anna.kalogirou@nottingham.ac.uk

D. Henry \& G. Thomas: Department of Applied Mathematics, University College Cork, Cork, Ireland; d.henry@ucc.ie \& g.thomas@ucc.ie

Digital Object Identifier https://doi.org/10.36688/imej.3.37-44 performed of water-wave impact in a channel with a V-shaped and closed contraction at its end. This work will be reviewed briefly, as well as the waterwave impact phenomenon, before demonstrating how it inspired the novel wave-energy device presented here.

First, in these shallow-layer flows, multiple throughflow states emerged under (quasi-)steady flow, including a subcritical reservoir flow state with much higher layer depths, typically four to six times higher than the depths in the fast supercritical flow state [1], [13]. Second, water-wave impact against sea walls involves impulsive forces and very high collapsing jets [14]. Third, these effects have been combined in the channel with a V-shaped end in which a series of solitons has been generated (a high one, an intermediate-height one and a low one) by a finite-time removal of a lock gate between two water levels at rest at the beginning of the channel. In the experiments, the first soliton travelled fastest and was about $0.35 \mathrm{~m}$ in height when it entered the V-shaped channel contraction. As it progressed, it broke into a hydraulic bore, diminished slightly in amplitude due to turbulent wave breaking and emerged as a smooth soliton again upon entering the contraction. It subsequently reflected in the V-shaped contraction, with minor amplification, to draw a deep trough in which the second unbroken soliton exactly crashed, to create a wave or jet of about $3.5 \mathrm{~m}$ in height. Hence, the abnormality index $A I$ used for rogue waves, defined as the rogue-wave height over the ambient wave height, was about $A I=10$ [2].

While our set-up has been engineered, it has relations with rogue-wave generation at sea, either midocean in crossing seas with two or even three main wave directions under a certain angle, or at the coast by incoming waves interacting with a V-shaped submerged or topographical convergence lying under as well as above the water surface. A simplified modelling environment for water waves in crossing seas or with crossing solitons consists of the KadomtsevPetviashvilli equation [6], [9], in which maximum amplifications of two or three solitons interacting under an optimal angle can lead to wave amplification of 4 to 8.6 times, using analytical solutions [5], [6].

Several existing wave-energy devices have been inspired by such wave-amplification phenomena, observed by crossing seas under mid-ocean conditions or by sea walls or topography-induced coastal convergences. These devices will be described below since they are related to our novel wave-energy device, referring to Falcão [4] for more details. First, in the wellknown TapChan device a natural convergence along 
the coastline is used to amplify the wave height. At the end of this natural convergence there is an engineered channel end which lets waves run over a man-made wall leading into a higher-level reservoir. Hydropower is used at the other end of the reservoir to generate electricity by making use of the difference in water levels. Second, oscillating water columns (OWCs) consist of an enclosed contracting cavity in which a rising or falling water surface compresses or decompresses air driving a Wells turbine placed in a nozzle at the end of the curved cavity for power generation. OWCs have been installed at the coast or on floating or anchored platforms in deeper waters. The IP wave buoy (IPWB) is a different type of device, in which one pipe segment has been anchored to the sea bottom to stay relatively fixed in the vertical direction, while an insert bobs up and down under the wave motion, thus generating energy via a power-take-off (PTO) device. The energy is often used in-situ to power the navigation lights placed on the buoy. Our novel wave-energy device is a deconstruction of the above three together with the Berkeley wedge [12], and a reconstruction using different aspects of the above four devices [2], [7], [8]. A proof-of-principle of the device has been built; this was tested in $2013^{1}$ and is shown in figure 1.

The device consists of a V-shaped contraction with vertical walls and a flat bottom, in which a simplexshaped wave-buoy is placed constrained to move in one direction only, either along a slight arc or along the vertical. It has a straight or curved mast, on top of which there are a series of magnets that move through induction coils. The movement of the wavebuoy by the incoming waves leads to magneticallyinduced power generation. In the sketch of figure 2a, a more robust version is shown (compared to that in figure $1 \mathrm{~b}$ ), wherein the buoy pivots around a point of rotation such that it can also be rotated out of the contraction during a storm.

The outline of the paper is as follows. In §II a complete mathematical model is given of the fully coupled nonlinear water-wave motion, the buoy motion and the induction motor, with the dissipative loads and losses in the electrical circuits involved. In an accompanying Appendix A, it is shown that the conservative dynamics follows from a variational principle. In §III the linearisation is introduced, with a compatible finiteelement discretisation stated in Appendix B, which is by construction fully consistent with the constraints involved. Simulation results using this discretisation are shown in $\S \mathrm{IV}$, followed by a discussion in $\S \mathrm{V}$.

\section{MAthematical MODEL OF THE DEVICE}

The complete mathematical model consists of three components:

- the nonlinear potential-flow water-wave motion in the wave tank and its contraction;

- the constrained buoy motion induced by the water waves; and,

${ }^{1}$ See https: / / www.youtube.com/watch?v=SZhe_SOxBWo\&t=254s and [2].

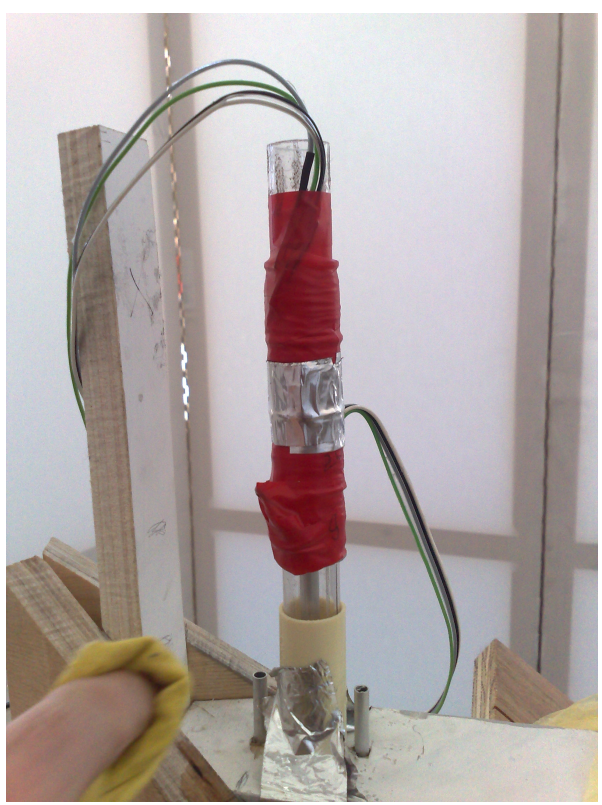

(a)

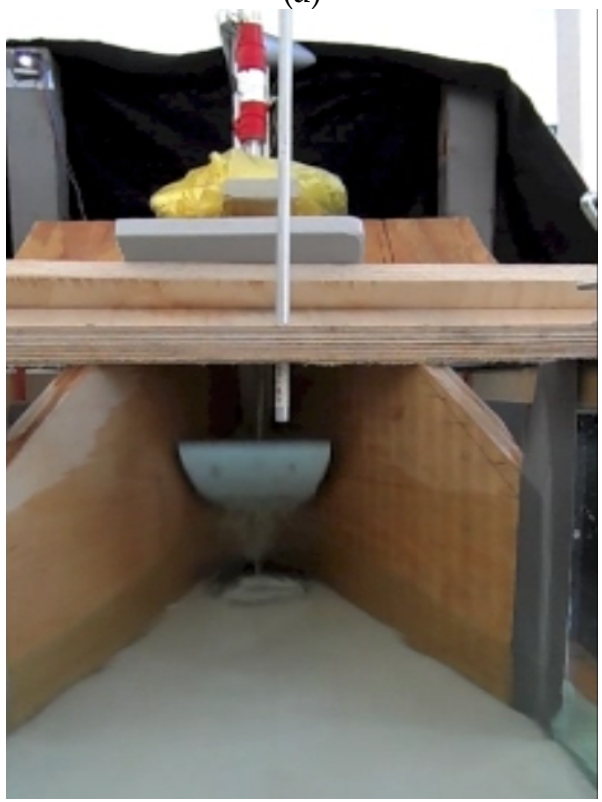

(b)

Fig. 1. A proof-of-principle of the scaled wave-energy device is shown: (a) a zoom-in of the electro-magnetic generator showing the hollow tube (covered in red tape) with its coils and the LED; the magnets on top of the buoy-mast move through the coils; and, (b) a view in the contraction with the buoy and a blinking LED light (top left).

- the power generated through electro-magnetic induction. This generator comprises the magnets attached to a mast on the buoy moving through fixed induction coils, and the loads consuming the power generated, here modelled as LEDs.

The wave tank ranges from a piston wavemaker at $y=R(t)$ up to the convergent point of the linear contraction at $y=L_{y}$. The contraction starts at $y=L_{y}-L_{c}$ and the width of the main channel is $L_{x}$, with the channel centre line at $x=L_{x} / 2$, cf. figure $2 \mathrm{~b}$. Hence, the wavetank has the horizontal extent $x \in\left[0, L_{x}\right]$ and $y \in\left[R(t), l_{y}(x)\right]$, with $l_{y}(x)=L_{y}-L_{c}\left|1-2 x / L_{x}\right|$.

The water waves are modelled using classical potential flow with a free surface. The velocity $\mathbf{u}$ of the 


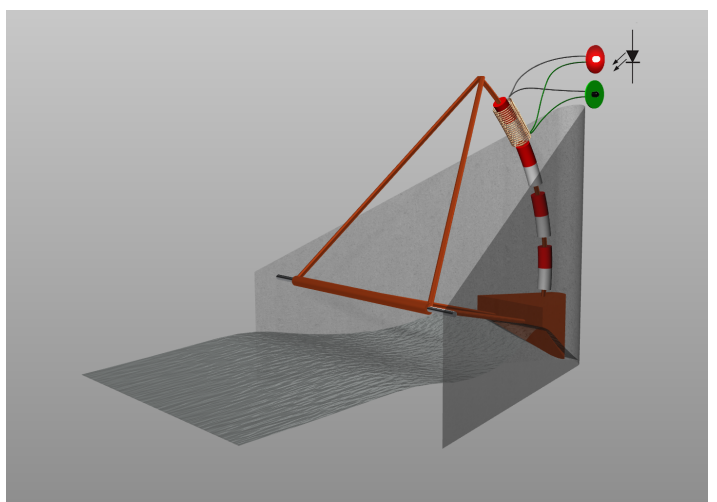

(a)

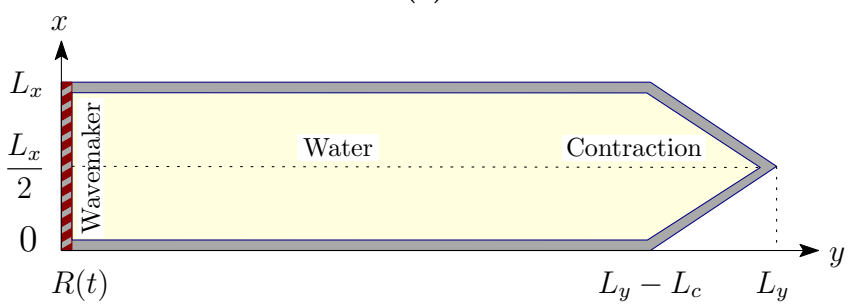

(b)

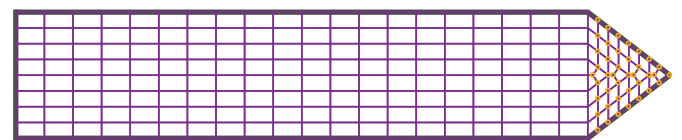

(c)

Fig. 2. (a) Sketch of our wave-energy device with its horizontal axle at the contraction entrance, and its three-dimensional tetrahedral buoy in the corner of the contraction. The buoy is attached to an induction motor, consisting of magnets on the arc moving through the hollow cylindrical coils indicated in yellow, while the LEDs are shown with green and red. (b) Top-view schematic of the waveenergy tank used in the simulations, and (c) the finite-element mesh.

water is approximated by $\mathbf{u}=\boldsymbol{\nabla} \phi$ using a velocity potential $\phi=\phi(x, y, z, t)$, with horizontal coordinates $x$ and $y$, vertical upward coordinate $z$ and time $t$. Gravity acts downwards with acceleration $g$. The bottom of the channel is horizontal at $z=0$ and the free surface lies at $z=h(x, y, t)$, with water depth $h(x, y, t)$, assuming a single-valued non-overturning free water surface. Luke's variational principle [11] can be used to model and derive the equations for three-dimensional waterwave motion, cf. [3].

The buoy is a tetrahedral simplex with a flat top face, two vertical faces aligned with the linear contraction walls, and a slanted face focussing in a point at the bottom, cf. [2]. The combination of buoy, its vertical mast and the magnets has an overall mass $M$ and vertical coordinate $Z=Z(t)$, whose rest position $\bar{Z}$ is determined by Archimedes' principle. The top of the mast above $Z$ is $H_{m}$, and $z=Z+H_{m}$ lies at the centre of the magnet. The vertical velocity of the buoy is $W=\dot{Z} \equiv \mathrm{d} Z / \mathrm{d} t$. The time-dependent position of the buoy is given by

$$
z=h_{b}(x, y ; Z(t))=Z(t)-H_{k}-\tan \alpha\left(y-L_{y}\right),
$$

with $\alpha$ a buoy angle and $H_{k}$ the depth of the buoy's keel such that $z=Z-H_{k}$ is at the apex or bottom point of the buoy at $y=L_{y}$. At rest, the depth in the main wavetank is $h=H_{0}$, while under the buoy it is $H(y)=h_{b}(y ; \bar{Z})$. The waterline at $y=y_{b}(x, t)$, here assumed to be single-valued in $y$, is located where the water depth just equals the buoy depth, i.e. where

$$
h\left(x, y_{b}(x, t), t\right)=h_{b}\left(x, y_{b}(x, t) ; Z(t)\right) .
$$

In general, (2) is an implicit relation defining the waterline $y_{b}$ but for the simplex-shaped buoy it is an explicit relation since $h_{b}$ is linear in $y$ and independent of $x$, even though the nonlinear waterline position $y_{b}(x, t)$ is a function of $x$ and $t$.

The electro-magnetic induction generator consists of a circuit with induction coils and inductance $L_{i}$, and a series of parallel circuits with LEDs as loads. The resistance of the inductance coils is $R_{c}$ and the resistance of the rest of the circuit is $R_{i}$. The induction coils have a radius $a$, length $L$ and $N$ turns. The magnetic dipole momentum of the magnet is $\mu$, the magnet's length is $L_{m}$ and its radius $A_{m}<a$ since the magnet can move freely through the coils. The magnet lies in the timedependent range $z \in\left[Z(t)+H_{m}-L_{m} / 2, Z(t)+H_{m}+\right.$ $\left.L_{m} / 2\right]$, while the induction coils lie in the fixed range $z \in\left[\bar{Z}+\left(1+\alpha_{h}\right) H_{m}-L / 2, \bar{Z}+\left(1+\alpha_{h}\right) H_{m}+L / 2\right]$, with $0<\alpha_{h}<1$.

Two sets of LEDs are circuited in parallel in opposite directions such that one set of LEDs is always operating under the AC-currents generated. The two unknowns in the model are the charge $Q=Q(t)$ and the current $I=I(t)=\dot{Q} \equiv \mathrm{d} Q / \mathrm{d} t$ through the circuit. A straightforward adaptation of Shockley's equation for LEDs then yields

$$
V_{s}(I)=-\operatorname{sign}(I) n_{q} V_{T} \ln \left(|I| / I_{\text {sat }}+1\right),
$$

with the $\operatorname{sign}(I)$-function and absolute value $|I|$ used to model each set of two LEDs in parallel. Here, $n_{q}$ is the quality factor, $V_{T}$ is the thermal voltage and $I_{\text {sat }}$ is the saturation current. Rather than modelling the current $I(t)$, the variational modelling puts priority to a variable $P_{Q}(t)$ conjugate to $Q(t)$. Due to the intrinsic coupling of the electro-magnetic generator and the buoy, $P_{Q}$ is then defined as follows [2]

$$
P_{Q}=L_{i} \dot{Q}-K(Z), \text { with } K(Z)=\int^{Z} \gamma G(\hat{Z}) \mathrm{d} \hat{Z}
$$

where $\gamma=2 \pi a^{2} \mu N / L$ and function $G(Z)$ is defined by

$$
G(Z)=\frac{1}{\pi A_{m}^{2} L_{m} a} \int_{-L / 2}^{L / 2} \int_{0}^{2 \pi} \int_{0}^{A_{m}} F(z) \mathrm{d} r \mathrm{~d} \theta \mathrm{d} q,
$$

with

$$
\begin{aligned}
F(z) & =f(-z)-f(z), \\
f(z) & =\frac{r(a-r \cos \theta)}{\left(r^{2}+\left(\frac{L_{m}}{2}+z\right)^{2}+a^{2}-2 r a \cos \theta\right)^{3 / 2}}, \\
z & =q+\bar{Z}+\alpha_{h} H_{m}-Z .
\end{aligned}
$$

Here we will consider an approximation of the function $G(Z)$, valid in the far field of the magnet and given by

$$
G_{\text {approx }}(Z) \approx \frac{1}{\left(a^{2}+\left(\bar{Z}+\alpha_{h} H_{m}-Z-\frac{L}{2}\right)^{2}\right)^{3 / 2}}
$$




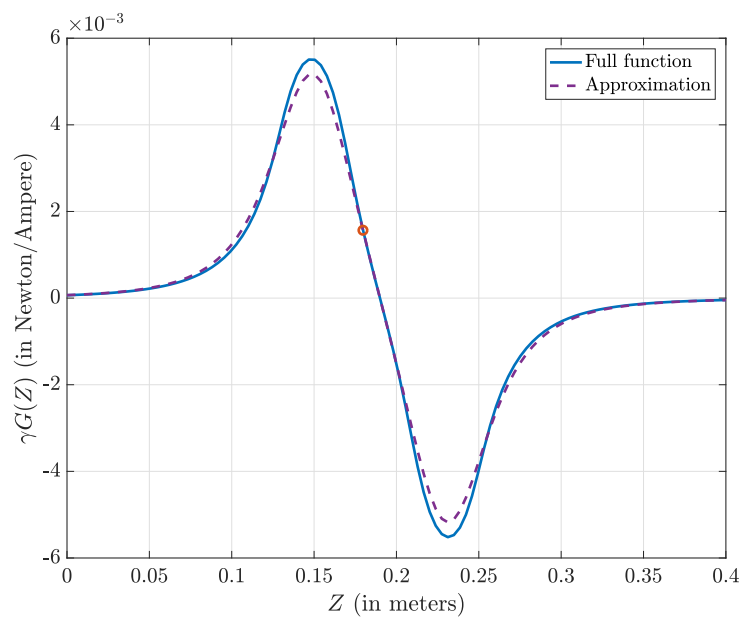

Fig. 3. Function $G(Z)$ (defined in (5)) and its approximation $G_{\text {approx }}(Z)$ in the far field of the magnet (defined in (6)). The circle denotes the value $G(\bar{Z})$ at the rest position $\bar{Z}=0.18$. The values used for the rest of the parameters are taken from the table found in the appendix of [2].

$$
-\frac{1}{\left(a^{2}+\left(\bar{Z}+\alpha_{h} H_{m}-Z+\frac{L}{2}\right)^{2}\right)^{3 / 2}} .
$$

A plot of $\gamma G(Z)$ for the full function in (5) and its approximation in (6) is shown in figure 3; the two functions clearly lie close to each other, especially at the rest position $\bar{Z}$ that will be used later (shown with a circle in figure 3), which confirms that the far-field approximation is appropriate in the present study.

The representation of the coupled system concerning the nonlinear water waves, the buoy motion and the electro-magnetically induced currents will be obtained using variational methods. In the absence of the dissipative effects caused by the resistance of electrical circuits, induction coils and LED loads, the dynamics can be derived from a single variational principle (VP), detailed in Appendix A. With each respective variation indicated, variations of this VP (17) then yield the following governing equations and boundary conditions,

$$
\begin{aligned}
& \delta \phi: \nabla^{2} \phi=0 \\
&\left.(\delta \phi)\right|_{z=h}: \partial_{t} h+\nabla_{H} \phi \cdot \nabla h-\partial_{z} \phi=0 \text { on } z=h, \\
& \delta h: \quad \partial_{t} \phi+\frac{1}{2}|\nabla \phi|^{2}+g\left(h-H_{0}\right) \\
&+\lambda \Theta\left(y-y_{b}\right)=0 \quad \text { on } \quad z=h, \\
& \delta \lambda: \quad h-h_{b}=0 \text { for } y \geq y_{b}, \\
& \delta W: \quad \dot{Z}=W \\
& \delta Z: \quad M \dot{W}=-M g-\gamma\left(P_{Q}+K(Z)\right) \frac{G(Z)}{L_{i}} \\
&+\rho_{0} \int_{0}^{L_{x}} \int_{0}^{l_{y}(x)} \lambda \Theta\left(y-y_{b}\right) \mathrm{d} x \mathrm{~d} y, \\
& \delta P_{Q}: \dot{Q}=\left(P_{Q}+K(Z)\right) / L_{i}, \\
& \delta Q: \dot{P}_{Q}=0
\end{aligned}
$$

with $\nabla_{H}=\left(\partial_{x}, \partial_{y}\right)^{T}, \boldsymbol{\nabla}=\left(\partial_{x}, \partial_{y}, \partial_{z}\right)^{T}$ and Heaviside function $\Theta\left(y-y_{b}\right)$ being nonzero/unity where its argument is positive, i.e. for $y>y_{b}(x, t)$, with $y_{b}(x, t)$ the waterline under the buoy. The Laplace equation (7a) describes the incompressible dynamics $\boldsymbol{\nabla} \cdot \mathbf{u}=$ $\boldsymbol{\nabla} \cdot \boldsymbol{\nabla} \phi=0$ of the water under the free surface, in terms of the velocity potential $\phi=\phi(x, y, z, t)$. The freesurface dynamics is governed by a kinematic equation (7b) for the water depth $h(x, y, t)$ as well as Bernoulli's equation (7c) for $\partial_{t} \phi$ or $\phi(x, y, h(x, y, t))$ evaluated at $z=h(x, y, t)$. The motion of the vertical position $Z(t)$ of the buoy is given by (7e) and its acceleration, progressing velocity $W(t)$, by (7f). The charge $Q(t)$ in the electrical circuit is governed by $(7 \mathrm{~g})$ and its conjugate momentum $P_{Q}(t)$, related to the current $I(t)$, by $(7 \mathrm{~h})$. Water-wave and buoy motion are coupled using a Lagrange multiplier $\lambda=\lambda(x, y, t)$ such that the shape $h(x, y, t)$ of the water surface is forced to equal the shape $h_{b}(x, y ; Z(t))$ of the buoy on the wetted surface of the buoy. It is shown in [2] that $\lambda$ is the pressure on the buoy; moreover, at the waterline this pressure is zero, i.e. $\lambda\left(x, y_{b}(x, t), t\right)=0$. Hence, it is only defined under the time-dependent wetted surface of the buoy, i.e. for $y>y_{b}(x, t)$. This coupling Lagrange multiplier appears in both the Bernoulli equation (7c) as well as the equation for the vertical acceleration (7f). In the latter equation, this integrated pressure on the buoy yields the force imposed by the water pressure on the buoy. Buoy motion and electro-magnetic currents are coupled through the connecting function $G(Z)$ defined in (5), which appears in the equation (7f) for the vertical acceleration. Note that the constrained equation $(7 \mathrm{~d})$ needs to be maintained over time to ensure consistency. This consistency issue will be analysed in a simplified model set-up in the next section.

Finally, when the resistance of the electrical circuit, the induction coils and the loads in (3) are added, then the last equation $(7 \mathrm{~h})$ is extended to the following

$$
\dot{P}_{Q}=-\left(R_{c}+R_{i}\right) I-\operatorname{sign}(I) n_{q} V_{T} \ln \left(\frac{|I|}{I_{s a t}}+1\right) .
$$

Given that $P_{Q}=L_{i} I-K(Z)$, its time derivative is $\dot{P}_{Q}=L_{i} \dot{I}-\gamma G(Z) \dot{Z}$ and hence (8) can be rewritten as

$$
\begin{aligned}
L_{i} \dot{I}=\gamma G(Z) \dot{Z} & -\left(R_{c}+R_{i}\right) I \\
& -\operatorname{sign}(I) n_{q} V_{T} \ln \left(|I| I_{\text {sat }}+1\right) .
\end{aligned}
$$

Equation (9) describes the change of the current in time; the electrical resistance terms and the loads are clearly seen to result in dissipation of the current.

\section{LINEARISATION AND DISCRETISATION}

Rather than considering the fully nonlinear system of equations, it was decided to first consider its linearisation in the shallow-water limit (see [2]), using the following decomposition of the variables into a basic state at rest and perturbations

$$
\begin{aligned}
\phi(x, y, z, t) & =\tilde{\phi}(x, y, z, t), \\
h(x, y, t) & =H(x, y)+\eta(x, y, t), \\
\lambda(x, y, t) & =\Lambda(y)+\tilde{\lambda}(x, y, t), \\
y_{b}(x, t) & =L_{b}+\tilde{y}_{b}(x, t), \\
Z(t) & =\bar{Z}+\tilde{Z}(t), \\
W(t) & =\tilde{W}(t), \\
Q(t) & =\tilde{Q}(t),
\end{aligned}
$$




$$
P_{Q}(t)=-K(\bar{Z})+\tilde{P}_{Q}(t)
$$

with $H(x, y)$ the rest-state depth, which is $H_{0}$ for the free surface-at-rest for $y<L_{b}$ and $H=H(y)$ under the buoy for $y>L_{b}$, and $\Lambda(y)$ the hydrostatic pressure under the buoy at rest.

Considering (9) for small loads, one notes that $\operatorname{sign}(I) n_{q} V_{T} \ln \left(|I| / I_{\text {sat }}+1\right) \approx n_{q} V_{T} I / I_{\text {sat }} \equiv R_{l} I$, with an effective resistance $R_{l}=n_{q} V_{T} / I_{\text {sat }}$ of the LED loads. When the change of $I$ in time is also small, such that $\dot{I} \approx 0$, one thus finds that

$$
\left(R_{c}+R_{i}+R_{l}\right) I \approx \gamma G(Z) \dot{Z} .
$$

Consequently, the vertical momentum equation (7f) for the buoy, mast and magnet approximately becomes

$$
\begin{aligned}
M \dot{W}=-M g & -\frac{\gamma^{2} G^{2}(Z)}{L_{i}\left(R_{c}+R_{i}+R_{l}\right)} W \\
& +\rho_{0} \int_{0}^{L_{x}} \int_{0}^{l_{y}(x)} \lambda \Theta\left(y-y_{b}\right) \mathrm{d} x \mathrm{~d} y .
\end{aligned}
$$

Hence, as expected, the resistance and loads combined are seen to act as a drag on the buoy-mast-magnet unit in this partial linear limit.

By only keeping terms to leading order in the perturbation and taking the depth-avaraged shallowwater limit, cf. [2], a simplified shallow-water system is obtained in which all fields are functions of the horizontal coordinates and time only. Hence, $\eta=\eta(x, y, t)$, $\phi=\phi(x, y, t)$ and $\lambda=\lambda(x, y, t)$. The gradient is likewise redefined as the horizontal operator $\boldsymbol{\nabla}=\left(\partial_{x}, \partial_{y}\right)^{T}$. The ensuing system becomes

$$
\begin{aligned}
& \eta-\tilde{Z}=0 \text { for } y \geq L_{b}, \\
& \dot{R}=\partial_{x} \tilde{\phi} \quad \text { at } \quad x=0 \text {, } \\
& \partial_{t} \eta+\boldsymbol{\nabla} \cdot(H \boldsymbol{\nabla} \tilde{\phi})=0 \text {, } \\
& \partial_{t} \tilde{\phi}+g \eta+\tilde{\lambda} \Theta\left(y-L_{b}\right)=0 \text {, } \\
& \dot{\tilde{Z}}=\tilde{W} \text {, } \\
& M \dot{\tilde{W}}+\gamma G(\bar{Z}) \frac{\left(\tilde{P}_{Q}+\gamma G(\bar{Z}) \tilde{Z}\right)}{L_{i}} \\
& -\rho_{0} \int_{0}^{L_{x}} \int_{0}^{l_{y}(x)} \tilde{\lambda} \Theta\left(y-L_{b}\right) \mathrm{d} y \mathrm{~d} x=0, \\
& \dot{\tilde{Q}}=\frac{\left(\tilde{P}_{Q}+\gamma G(\bar{Z}) \tilde{Z}\right)}{L_{i}}, \\
& \dot{\tilde{P}}_{Q}=-\left(R_{c}+R_{i}+R_{l}\right) \frac{\left(\tilde{P}_{Q}+\gamma G(\bar{Z}) \tilde{Z}\right)}{L_{i}}, \\
& \boldsymbol{\nabla} \cdot(H \nabla \tilde{\lambda})-\frac{\rho_{0}}{M} \int_{0}^{L_{x}} \int_{0}^{l_{y}(x)} \tilde{\lambda} \Theta\left(y-L_{b}\right) \mathrm{d} y \mathrm{~d} x= \\
& -\nabla \cdot(g H \nabla \eta)-\frac{\gamma}{M} G(\bar{Z}) \tilde{I} \quad \text { for } \quad y \geq L_{b},
\end{aligned}
$$

where a consistency requirement has been added which arises by taking twice the time derivative of the constraint $\eta-\tilde{Z}=0$, (13a), while using other equations in (13) to eliminate the time derivatives. It leads to the elliptic equation (13i) for the Lagrange multiplier $\tilde{\lambda}$, the perturbation pressure under the buoy. Now one can either solve the system (13) excluding the last consistency equation (13i), or solve the system
(13) excluding the first constrained equation (13a). Otherwise, the system (13) is seen to be overspecified. In either case, it can be shown that one subset is and has to be equivalent to the other subset. Note also that the linearised effect of a piston wavemaker is added at $x=0$, with $\dot{R}$ driving the wave motion.

A consistent discretisation in space and time needs to mimic the consistency requirement discussed above. A continuous $C_{0}$-Galerkin finite-element discretisation has been used in space with standard linear tent functions of second-order accuracy, with an extra trick. To ensure consistency in time, it was necessary to weakly extend the basis function at $y=L_{b}$, the linearised waterline position, into $y<L_{b}$. Otherwise, it is as yet unclear how to obtain a stable and consistent time discretisation. A consistent time discretisation now involves a combination of forward and backward Euler discretisations in time, essentially forming a constrained symplectic-Euler time discretisation, cf. [10]. The final space-time discretisation is given in Appendix B.

\section{Results}

The space-time discrete system of linear equations (18) in Appendix B has been solved using an inhouse code developed by employing the finite-element method described in the previous section. The numerical domain uses $L_{x}=0.2 \mathrm{~m}$ and $L_{y}=2 \mathrm{~m}$, corresponding to a physical domain of horizontal extent $L_{x} \times L_{y}$. The water depth at rest is taken to be $H_{0}=0.2$ $\mathrm{m}$ and the buoy-mast-magnet unit was considered to have a total mass of $M=0.08 \mathrm{~kg}$. Also defined is a computational grid consisting of $N_{x}=10$ and $N_{y}=50$ elements in the $x$ - and $y$-directions, respectively. A sample coarse-resolution grid can be seen in figure 2c, with yellow marks denoting the nodes in the contraction.

The initial, rest state is disturbed by the motion of the piston wavemaker at one wall of the wavetank. The wavemaker has amplitude $A=0.0616 \mathrm{~m}$ and frequency $\omega=8.8 \mathrm{~s}^{-1}$. The generated waves propagate along the channel and take approximately 1.3 seconds to reach the contraction and impact upon the waveenergy device. They are then amplified along the wall of the contraction and obtain a maximum amplification in the corner, simultaneously forcing the buoy to shift upwards. Representative snapshots of the simulation at different times can be seen in figure 4 .

Various quantities are tracked as simulation outputs, including the wave profile, velocity potential, vertical displacement and velocity of the buoy, as well as the electrical current, voltage and power generated due to the motion of the magnet through the coils as a result of the wave impact on the buoy. In the linearised limit considered here, the (mean) total power generated $\tilde{P}_{g}$ and the (mean) power lost $\tilde{P}_{l}$ are determined over the course of one simulation for $0 \leq t \leq T$, as follows

$$
\left[\tilde{P}_{g}\right]=\frac{1}{T} \int_{0}^{T} \tilde{P}_{g}(t) \mathrm{d} t \text { and }\left[\tilde{P}_{l}\right]=\frac{1}{T} \int_{0}^{T} \tilde{P}_{l}(t) \mathrm{d} t
$$

with $\tilde{P}_{g}(t)=R_{l} \tilde{I}^{2}(t)$ and $\tilde{P}_{l}(t)=\left(R_{c}+R_{i}\right) \tilde{I}^{2}(t)$. In figure 5 , some of these outputs are shown and 

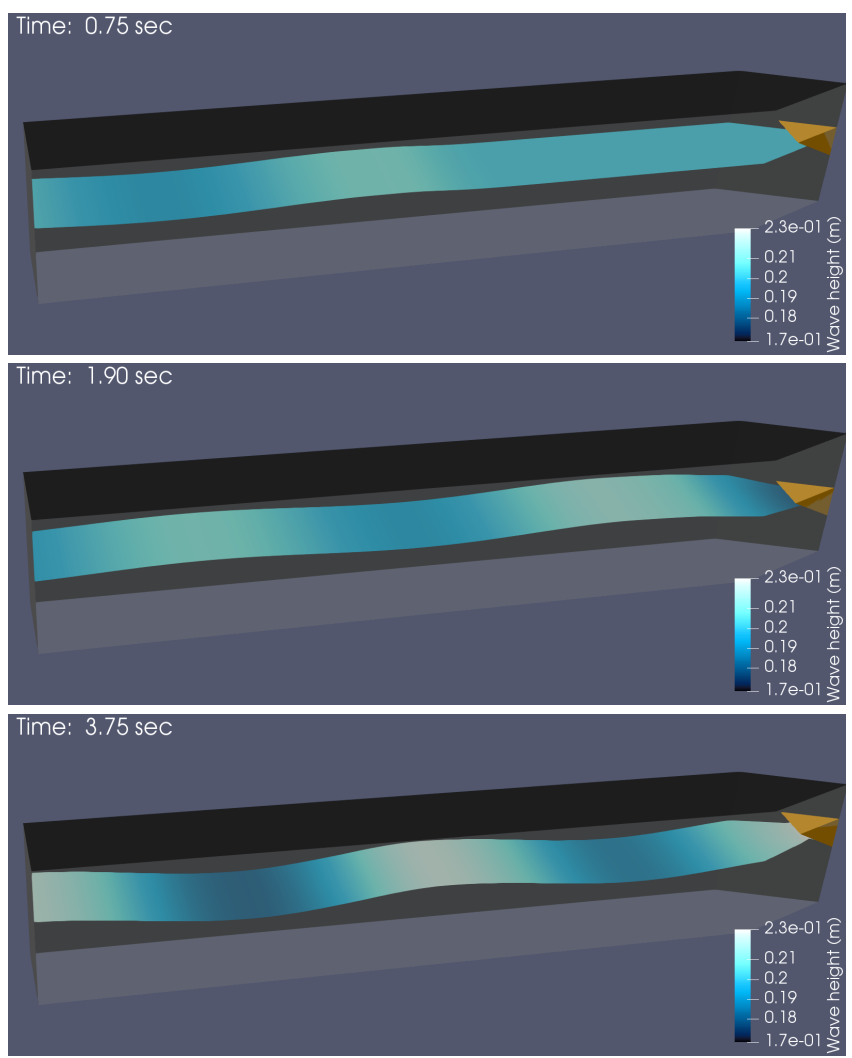

Fig. 4. Snapshots of the numerically computed free surface $h(x, y, t)=H(x, y)+\eta(x, y, t)$ (colour denotes height in meters, with initial still water level $\left.H_{0}=0.2 \mathrm{~m}\right)$, as it evolves in the wavetank with V-shaped contraction (times shown on the pictures). The buoy resides in the corner of the contraction and is shown using orange colour.

are illustrating the motion of the buoy, the power generation and the losses in the circuit. In particular, it can be seen that once the waves reach the contraction at around 1.3 seconds, then the wave-energy device follows an oscillatory motion in response to the incoming waves. The oscillating motion of the buoy and the resulting movement of the magnet, which is attached on a mast on top of the buoy, through the induction coils, generates current and hence produces power. Part of the generated power is lost due to circuit resistance and the rest is used to illuminate one of the LEDs. The effect of wavemaker amplitude $A$ and frequency $\omega$ on the amount of total (mean) power generated or lost (calculated through (14)) was further investigated and is demonstrated in figure 6 . For the frequencies and amplitudes tested here, it can be seen that the power increases monotonically with the wavemaker amplitude, while increasing the frequency leads to a non-monotonic behaviour in the power generation/loss. The mean power generated $\left[\tilde{P}_{g}\right]$ scales like $0.00124 A^{2}$. The optimal frequency, for which the maximum amount of power is generated, is observed to be $\omega_{c} \approx 15.4 \mathrm{~s}^{-1}$. Further tests are required to determine whether this critical frequency $\omega_{c}$ is related to the resonant frequency of the setup employed here.
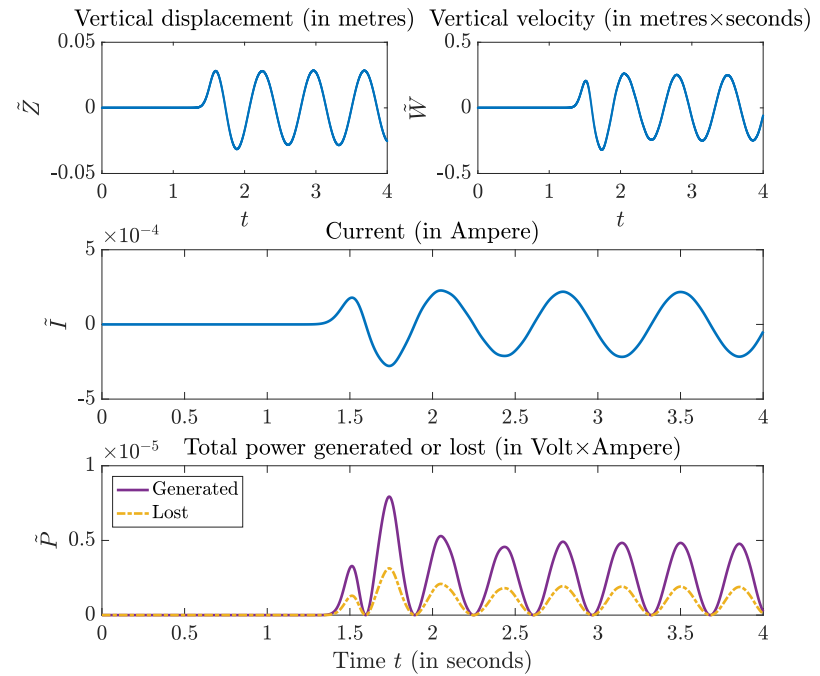

Fig. 5. Output of the numerical simulation demonstrating the response of the wave-energy device to the incoming waves (panels on top row; left: vertical displacement, right: velocity). The energy generated is also shown in terms of current (middle panel) and power (bottom panel). The power output depicted in the bottom panel is divided into two parts: the power output to the LEDs (solid purple line) and the power lost in the electrical circuit (dash-dotted yellow line)
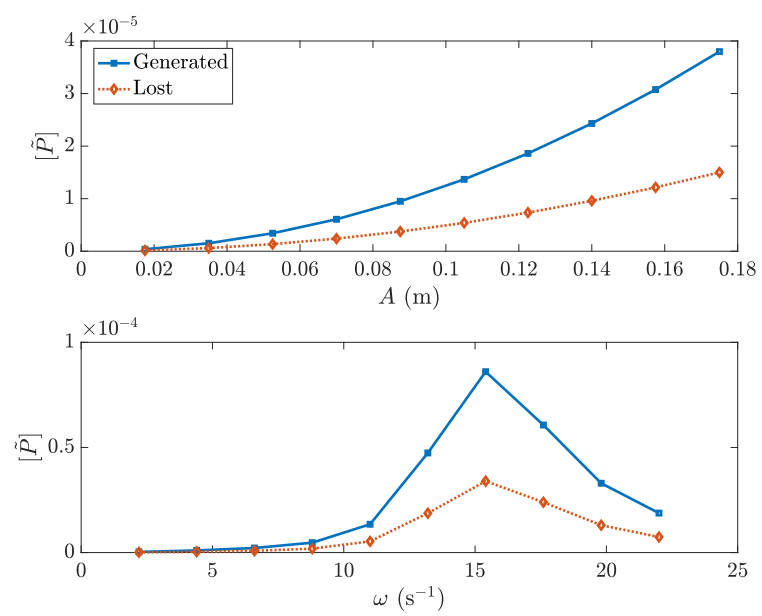

Fig. 6. Variation of the total mean power generated (in Volt $\times$ Ampere) against the wavemaker amplitude or frequency. The mean power is calculated from (14) over the duration of one simulation with $T=10 \mathrm{~s}$. Top panel: varied wavemaker amplitude $A$ (in m) with fixed frequency $\omega=8.8 \mathrm{~s}^{-1}$. Bottom panel: varied wavemaker frequency $\omega$ (in $s^{-1}$ ) with fixed amplitude $A=0.0616 \mathrm{~m}$.

\section{DISCUSSION}

The innovative wave-energy device presented in this paper was developed following the observation of wave-amplification phenomena realised in boresoliton-splash experiments, described in [2]. This device is targeted primarily for placement in a breakwater where incident wave conditions are most suitable. Amongst the principal novel features are the use of a contraction to enhance the wave-buoy motion and magnetically-induced energy generation. A laboratory proof-of principle shows that the wave-energy device operates in a satisfactory manner.

A fully nonlinear wave-to-wire mathematical model has been developed for the combined (potential-flow) 
hydrodynamics, the buoy motion and the power generation by magnetic induction. An important building block in the construction of the model is the utilisation of a single variational principle, from which the equations of motion emerge. A finite-element discretisation for both the simplified and linearised versions of the resulting nonlinear system has been formulated; this yields a consistent space-time discrete system of equations. The model also identifies the losses in power generation.

Preliminary numerical simulations of the linearised wave-energy system have been presented, demonstrating the motion of the waves, the response of the waveenergy device and the generation of energy. It has been found that the total amount of power generated increases monotonically for higher wavemaker amplitudes. However, this finding does not hold for increasing wavemaker frequencies and it was found that the generation of power increases only up to a critical frequency $\omega_{c}$, beyond which the power generation decreases. This latter finding is very similar to the establishment of a resonant frequency at $\omega_{c}$ and lying at the peak of a bandwidth curve but without the $a$ priori determination of these quantities.

Future work plans include the development and implementation of a fully nonlinear numerical model, the performance of complementary laboratory experiments for comparison and further simulations for validation purposes. Optimisation studies are planned to improve device performance. Initially these will concentrate upon the geometrical features of the contraction, such as angle and length, and the shape and weight of the wave buoy. Parallel studies will focus upon the importance of the resource, which will be associated with the wavemaker amplitude and wave frequency, and the number of loads and induction coils. When these component optimisations have been completed and understood, then an attempt to tackle the very difficult problem of complete device optimisation will be considered.

\section{REFERENCES}

[1] B. Akers and O. Bokhove 2008: Hydraulic flow through a contraction: multiple steady states. Phys. Fluids 20, 056601.

[2] O. Bokhove, A. Kalogirou and W. Zweers 2019: From boresoliton-splash to rogue waves, a new wave-energy device and extreme tsunami run-up. Water Waves 1, 217-258.

[3] C. Cotter and O. Bokhove 2010: Variational water-wave model with accurate dispersion and vertical vorticity. J. Eng. Math. 67, 33-54.

[4] A.F.O. Falcão 2010: Wave energy utilization: A review of the technologies. Renewable and Sustainable Energy Reviews 14, 899918 .

[5] F. Gidel, O. Bokhove and A. Kalogirou 2017: Variational modelling of extreme waves through oblique interaction of solitary waves: application to Mach reflection. Nonlinear Proc. Geoph. 24, 43-60.

[6] F. Gidel 2018: Variational water-wave models and pyramidal freak waves. PhD Thesis, University of Leeds, UK.

[7] A. Kalogirou and O. Bokhove 2016: Mathematical and numerical modelling of wave impact on wave-energy buoys. Proc. 35th Int. Conf. on Ocean, Offshore and Arctic Eng. (OMAE2016).

[8] A. Kalogirou, O. Bokhove and D. Ham 2017: Modelling of nonlinear wave-buoy dynamics using constrained variational methods. Proc. 36th Int. Conf. on Offshore Mechanics and Arctic Engineering (OMAE2017).

[9] Y. Kodama 2010: KP solitons in shallow water. Journal of Physics A: Mathematical and Theoretical 43, 434004
[10] B. Leimkuhler and S. Reich 2010: Simulating Hamiltonian Dynamics. Cambridge University Press. 379 pp.

[11] J. Luke 1967: A variational principle for a fluid with a free surface. J. Fluid Mech. 27, 395-397.

[12] F. Madhi and R.W. Yeung 2018: On survivability of asymmetric wave-energy converters in extreme waves. Renewable Energy 119, 891-909.

[13] A.W. Vreman, M. Al-Tarazi, J.A.M. Kuipers, M. van Sint Annaland, and O. Bokhove 2007: Supercritical shallow granular flow through a contraction: experiment, theory and simulation. J. Fluid Mech. 578, 233-269.

[14] D.H. Peregrine 2003: Water-wave impact on walls. Annu. Rev. Fluid Mech. 35, 23-43.

\section{APPENDIX A \\ VARIATION PRINCIPLE}

In the absence of any losses in the electrical circuits and without any loads, i.e. in the limits $R_{c} \rightarrow 0, R_{i} \rightarrow 0$ and $V_{T} \rightarrow 0$, the dynamics of the water waves, buoy motion and the electrical currents are succinctly described via one variational principle (VP). This VP is the sum of the three VPs of the three separate subsystems, augmented with the underlined term coupling the dynamics of the water waves to the buoy as well as the coupling terms between the buoy and the generator involving $K(Z)$ (or $G(Z)$ ). The VP reads

$$
\begin{aligned}
0=\delta & \int_{0}^{T} \mathcal{L}\left[\phi, h, Z, W, Q, P_{Q}, \lambda\right] \mathrm{d} t \\
\equiv \delta & \int_{0}^{T} \rho_{0} \int_{0}^{L_{x}} \int_{0}^{l_{y}(x)} \int_{0}^{h(x, y, t)} \partial_{t} \phi \mathrm{d} z \mathrm{~d} y \mathrm{~d} x \\
& \quad-M W \dot{Z}-P_{Q} \dot{Q}+\mathcal{H} \mathrm{d} t \\
\equiv & \int_{0}^{T} \rho_{0} \int_{0}^{L_{x}} \int_{0}^{l_{y}(x)} \int_{0}^{h(x, y, t)} \partial_{t} \phi+\frac{1}{2}|\nabla \phi|^{2} \\
& +g\left(z-H_{0}\right) \mathrm{d} z \mathrm{~d} y \mathrm{~d} x \\
& +\rho_{0} \int_{0}^{L_{x}} \int_{0}^{l_{y}(x)} \lambda\left(h-h_{b}\right) \Theta\left(y-y_{b}\right) \mathrm{d} x \mathrm{~d} y \\
& \quad-M W \dot{Z}-P_{Q} \dot{Q}+\frac{1}{2} M W^{2} \\
& +M g Z+\frac{1}{2} \frac{\left(P_{Q}+K(Z)\right)^{2}}{L_{i}} \mathrm{~d} t,
\end{aligned}
$$

in which a Lagrange multiplier $\lambda=\lambda(x, y, t)$ is used imposing that the shape of the free surface equals the dynamic shape of the buoy, thus imposing that $h-h_{b}=0$ weakly [7] (underlined in (15)). Furthermore, an extension of Luke's VP has been derived in [2] to show that the Lagrange multiplier $\lambda$ is the pressure under the buoy and that, consequently, at the waterline the Lagrange multiplier $\lambda\left(x, y_{b}, t\right)=0$, by taking the pressure above the free surface to be zero. Note that the energy $\mathcal{H}$ defined in the VP (15) consists of respective "kinetic" and "potential" energies of the water, buoy and the electro-magnetic induction generator plus one constraint. Here, the wavemaker $R(t)=0$ and its contribution was added in the linearised shallow-water model used in the simulations.

If the vector of unknowns is defined by $\mathbf{U}=$ $\left(\phi, h, Z, W, Q, P_{Q}, \lambda\right)^{T}$, with transpose $(\cdot)^{T}$, then variations of (15) are defined as follows

$$
\delta \int_{0}^{T} \mathcal{L}[\mathbf{U}] \mathrm{d} t=\lim _{\epsilon \rightarrow 0} \int_{0}^{T} \frac{(\mathcal{L}[\mathbf{U}+\epsilon \mathbf{U}]-\mathcal{L}[\mathbf{U}])}{\epsilon} \mathrm{d} t,
$$


with Lagrangian density $\mathcal{L}$ comprised of functions depending only on time, and fields depending on space and time. Variation of (15) yields

$$
\begin{aligned}
0= & \delta \int_{0}^{T} \mathcal{L}\left[\phi, h, Z, W, Q, P_{Q}, \lambda\right] \mathrm{d} t \\
= & \int_{0}^{T} \rho_{0} \int_{0}^{L_{x}} \int_{0}^{l_{y}(x)} \int_{0}^{h(x, y, t)} \underline{\partial_{t} \delta \phi+\nabla \phi \cdot \nabla \delta \phi} \mathrm{d} x \mathrm{~d} y \mathrm{~d} z \\
& +\rho_{0} \int_{0}^{L_{x}} \int_{0}^{l_{y}(x)}\left(\partial_{t} \phi+\frac{1}{2}|\nabla \phi|^{2}+g\left(z-H_{0}\right)\right. \\
& \left.\quad+\lambda \Theta\left(y-y_{b}\right)\right)\left.\right|_{z=h} \delta h \\
& \quad+\left(h-h_{b}\right) \Theta\left(y-y_{b}\right) \delta \lambda \mathrm{d} x \mathrm{~d} y \\
& +M(W-\dot{Z}) \delta W-P_{Q} \underline{\delta \dot{Q}} \\
& -\rho_{0} \int_{0}^{L_{x}} \int_{0}^{l_{y}(x)} \lambda \Theta\left(y-y_{b}\right) \mathrm{d} x \mathrm{~d} y \delta Z \\
& +\left(-M W \underline{\delta \dot{Z}}+M g+\gamma\left(P_{Q}+K(Z)\right) \frac{G(Z)}{L_{i}} \delta Z\right) \\
& +\left(-\dot{Q}+\left(P_{Q}+K(Z)\right) / L_{i}\right) \delta P_{Q} \mathrm{~d} t .
\end{aligned}
$$

Herein, end-point conditions $\delta \phi=\delta Q=\delta Z=0$ at $t=0, T$ have been used. The underlined terms require further manipulation using Gauss' law and integration by parts in time. Integration by parts in time involves dealing with the time-dependent location of the free surface $z=h(x, y, t)$. Gauss' law yields a contribution at the free surface $z=h(x, y, t)$ with its outward normal $\hat{\mathbf{n}}=(-\boldsymbol{\nabla} h, 1)^{T} / \sqrt{1+|\boldsymbol{\nabla} h|^{2}}$ and free surface element $d A=\sqrt{1+|\nabla h|^{2}} \mathrm{~d} x \mathrm{~d} y$. In addition, the variation of the Heaviside function has cancelled under the imposed condition $\lambda\left(y_{b}, t\right)=0$. These manipulations of (17a) lead to the final result

$$
\begin{aligned}
0= & \int_{0}^{T} \rho_{0} \int_{0}^{L_{x}} \int_{0}^{l_{y}(x)} \int_{0}^{h(x, y, t)}-\nabla \cdot(\nabla \phi) \delta \phi \mathrm{d} x \mathrm{~d} y \mathrm{~d} z \\
+ & \rho_{0} \int_{0}^{L_{x}} \int_{0}^{l_{y}(x)}\left(\partial_{t} \phi+\frac{1}{2}|\nabla \phi|^{2}+g\left(z-H_{0}\right)\right. \\
& \left.\quad+\lambda \Theta\left(y-y_{b}\right)\right)\left.\right|_{z=h} \delta h \\
& \quad+\left(\partial_{z} \phi-\partial_{t} h-\nabla h \cdot \nabla_{H} \phi\right)_{z=h}(\delta \phi)_{z=h} \\
& \quad+\left(h-h_{b}\right) \Theta\left(y-y_{b}\right) \delta \lambda \mathrm{d} x \mathrm{~d} y \\
+ & M(W-\dot{Z}) \delta W+\dot{P}_{Q} \delta Q \\
+ & \left(M \dot{W}+M g+\gamma\left(P_{Q}+K(Z)\right) \frac{G(Z)}{L_{i}}\right. \\
& \left.\quad-\rho_{0} \int_{0}^{L_{x}} \int_{0}^{l_{y}(x)} \lambda \Theta\left(y-y_{b}\right) \mathrm{d} x \mathrm{~d} y\right) \delta Z \\
+ & \left(-\dot{Q}+\left(P_{Q}+K(Z)\right) / L_{i}\right) \delta P_{Q} \mathrm{~d} t,
\end{aligned}
$$

with the system of equations (7) following because the variations $\delta h$ etc. are arbitrary.

\section{APPENDIX B}

\section{COMPATIBLE, CONSISTENT NUMERICAL DISCRETISATION}

Given the space-time discretisation procedure outlined in the main text and [2], the final space-time discrete system corresponding to (13) (not provided in
[2]) becomes the following

$$
\begin{aligned}
N_{\tilde{k} l}^{T}\left(\eta_{l}^{n+1}-1_{l} \tilde{Z}^{n+1}\right) & =0, \\
M_{k l} \frac{\left(\phi_{l}^{n+1}-\phi_{l}^{n}\right)}{\Delta t} & =-g M_{k l} \eta_{l}^{n}-N_{k \hat{l}} \lambda_{\hat{l}}^{n}-N_{k \tilde{b}} \lambda_{\tilde{b}}^{n}, \\
M_{k l} \frac{\left(\eta_{l}^{n+1}-\eta_{l}^{n}\right)}{\Delta t} & =S_{k l} \phi_{l}^{n}+T_{k} \dot{R}, \\
\frac{\left(\tilde{W}^{n+1}-\tilde{W}^{n}\right)}{\Delta t} & =C_{0} \tilde{Q}_{\hat{l}} \lambda_{\hat{l}}+C_{0} \tilde{Q}_{\tilde{b}} \lambda_{\tilde{b}}^{n} \\
& -C_{1} G(\bar{Z})\left(\tilde{P}_{Q}^{n}+\gamma G(\bar{Z}) \tilde{Z}^{n}\right) \\
\frac{\left(\tilde{Z}^{n+1}-\tilde{Z}^{n}\right)}{\Delta t} & =\tilde{W}^{n+1}, \\
\frac{\left(\tilde{Q}^{n+1}-\tilde{Q}^{n}\right)}{\Delta t} & =\frac{\left(\tilde{P}_{Q}^{n+1}+\gamma G(\bar{Z}) \tilde{Z}^{n}\right)}{L_{i}} \\
\frac{\left(\tilde{P}_{Q}^{n+1}-\tilde{P}_{Q}^{n}\right)}{\Delta t} & =-C_{2}\left(\tilde{P}_{Q}^{n+1}+\gamma G(\bar{Z}) \tilde{Z}^{n}\right), \\
\left(\tilde{S}_{\tilde{k} \hat{l}}+C_{0} \tilde{Q}_{\tilde{k}} \tilde{Q}_{\hat{l}}\right) \lambda_{\hat{l}}^{n} & =-g S_{\tilde{k} l} \eta_{l}-C_{0} \tilde{Q}_{\tilde{k}} \tilde{Q}_{\tilde{b}} \lambda_{\tilde{b}} \\
-\tilde{S}_{\tilde{k} \tilde{b}} \lambda_{\tilde{b}} & +C_{1} \tilde{Q}_{\tilde{k}} G(\bar{Z})\left(\tilde{P}_{Q}^{n}+\gamma G(\bar{Z}) \tilde{Z}^{n}\right), \\
\lambda_{\tilde{b}}^{n} & =g\left(\eta_{\tilde{b}}^{n}-\tilde{Z}^{n}\right),
\end{aligned}
$$

with abbreviating constants $C_{0}=\rho_{0} / M, C_{1}=$ $\gamma /\left(M L_{i}\right), C_{2}=\left(R_{c}+R_{i}+R_{l}\right) / L_{i}$ as well as mass and "Laplace" matrices [2]

$$
\begin{aligned}
M_{k l} & =\int_{0}^{L_{x}} \int_{0}^{l_{y}(x)} \varphi_{k}(x, y) \varphi_{l}(x, y) \mathrm{d} x \mathrm{~d} y \\
S_{k l} & =\int_{0}^{L_{x}} \int_{0}^{l_{y}(x)} H(y) \nabla \varphi_{k}(x, y) \cdot \nabla \varphi_{l}(x, y) \mathrm{d} x \mathrm{~d} y, \\
\tilde{S}_{\tilde{k} \tilde{l}} & =\int_{0}^{L_{x}} \int_{0}^{l_{y}(x)} H(y) \nabla \varphi_{\tilde{k}}(x, y) \cdot \nabla \varphi_{\tilde{l}}(x, y) \mathrm{d} x \mathrm{~d} y, \\
\tilde{Q}_{\tilde{k}} & =\int_{0}^{L_{x}} \int_{0}^{l_{y}(x)} \varphi_{\tilde{k}}(x, y) \mathrm{d} x \mathrm{~d} y \\
N_{k \tilde{l}} & =\int_{0}^{L_{x}} \int_{0}^{l_{y}(x)} \varphi_{k}(x, y) \varphi_{\tilde{l}}(x, y) \mathrm{d} x \mathrm{~d} y \\
T_{k} & =\int_{0}^{L_{x}} H(0) \varphi_{k}(0, y) \mathrm{d} y,
\end{aligned}
$$

with finite-element tent-shaped, test and basis, Galerkin functions $\varphi_{k}(x, y)$. In the entire horizontal domain, nodal indices $k, l$ are used, while nodal indices under the buoy are denoted by $\tilde{k}, \tilde{l}$ and indices of the finite-element nodes lying on the linearised waterline $y=L_{b}$ are denoted by $\tilde{b}$.

\section{ACKNOWLEDGEMENT}

OB thanks Dr Paul Steenson and Prof Steve Tobias for assistance with the derivation of the model for the electro-magnetic power generation. 